\title{
Transgenerational programming of maternal behaviour by prenatal stress
}

\author{
Isaac D Ward ${ }^{1}$, Fabíola C R Zucchi ${ }^{1}$, Jerrah C Robbins ${ }^{1}$, Erin A Falkenberg ${ }^{1}$, David M Olson², Karen Benzies ${ }^{3}$, \\ Gerlinde A Metz $^{1 *}$
}

\begin{abstract}
Peripartum events hold the potential to have dramatic effects in the programming of physiology and behaviour of offspring and possibly subsequent generations. Here we have characterized transgenerational changes in rat maternal behaviour as a function of gestational and prenatal stress. Pregnant dams of the parental generation were exposed to stress from days 12-18 (FO-S). Their daughters and grand-daughters were either stressed (F1-SS, F2-SSS) or non-stressed (F1-SN, F2-SNN). Maternal antepartum behaviours were analyzed at a time when pregnant dams usually show a high frequency of tail chasing behaviours. F1-SS, F2-SNN and F2-SSS groups showed a significant reduction in tail chasing behaviours when compared with controls. The effects of multigenerational stress (SSS) slightly exceeded those of transgenerational stress (SNN) and resulted in absence of tail chasing behaviour. These findings suggest that antepartum maternal behaviour in rats is programmed by transgenerational inheritance of stress responses. Thus, altered antepartum maternal behaviour may serve as an indicator of an activated stress response during gestation.
\end{abstract}

\section{Introduction}

The perinatal period is a time of high vulnerability to environmental influences. It is well established that maternal stress [1] and the quality of maternal care [2,3] influence offspring development and stress responses with consequences potentially lasting to adulthood [4-6]. Notably, maternal care is affected by stress during early post-natal development [7] as well as by stress during gestation [8].

Both stress and maternal care have been reported to program physiology and behaviour across generations $[4,9]$. Transgenerational programming of stress responses and associated trait anxiety were suggested to transmit to subsequent generations in the absence of stress via germ line-dependent mechanisms [10]. Non-genomic transmission of behavioural traits was also shown for maternal care in Long-Evans rats. Maternal care of offspring was highly correlated to the behaviour exhibited by their own mothers in the first week after parturition $[4,11,12]$. It was suggested that maternal stress and the mother's care determine offspring behavioural traits and their stress responses through epigenetic mechanisms [4,10,13-15].

\footnotetext{
* Correspondence: gerlinde.metz@uleth.ca

${ }^{1}$ Canadian Centre for Behavioural Neuroscience, University of Lethbridge,

4401 University Drive, Lethbridge, AB, Canada T1K3M4

Full list of author information is available at the end of the article
}

The epigenetic imprinting of adult physiology and behaviour by stress and maternal care suggests that this reciprocal relationship represents a potential target for prevention and intervention to improve offspring health outcomes.

The purpose of this study was to characterize changes in maternal behaviour as a function of transgenerational stress in rats. Antepartum maternal behaviour may offer predictive value as an indicator of an activated stress response and postpartum maternal care. Here we identified changes in maternal tail chasing as a sensitive measure of stress in antepartum maternal behaviour. Ancestral experience may determine the quality of maternal tail chasing and physiological responses to stress in the progeny. We hypothesized that antepartum maternal behaviour is programmed by transgenerational inheritance of stress response and associated with characteristic behavioural change. We compare the effects of gestational stress in the parental generation with the effects of programming by prenatal stress in subsequent generations. Our findings indicate that a reduction in tail chasing behaviour in pregnant dams prior to parturition is reflective of transgenerational programming by stress. 


\section{Methods}

\section{Animals}

Twenty-nine female adult Long-Evans Hooded rats, raised at the University of Lethbridge vivarium, were used. Eighteen non-stressed young adult males were used for breeding. During the test period, the pregnant dams were housed individually in standard polycarbonate shoebox cages (45.5 X 25.5 X $20 \mathrm{~cm}$ ) on corn cob bedding (Bed O Cobs 1 / 8“' The Andersons Lab Bedding, Ohio, USA). Maternal weight gain during pregnancy and litter size were monitored. The light cycle was 12:12 h with lights on at 07:30 h. The housing room was maintained at a temperature of $20^{\circ} \mathrm{C}$ and $30 \%$ relative humidity. The experiments were approved by the University of Lethbridge Animal Welfare Committee (protocol \#0803) according to guidelines set forth by the Canadian Council of Animals Care.

\section{Experimental design}

Three generations of rats were bred (see Figure 1) and subdivided into five groups: (1) Non-stressed controls $(\mathrm{n}=7)$; (2) Parental generation stressed during gestation (F0-S; n=6); (3) Prenatally stressed first filial generation that underwent gestational stress (F1-SS; $n=5)$; (4) Second filial generation that originated from a stressed parental generation, but neither they or their mothers were stressed (F2-SNN; n=5); (5) Second gestationally stressed filial generation that received prenatal stress through two previous generations (F2-SSS; $n=6)$. Thus, SNN rats represent a transgenerational condition of stress, while SSS rats represent a multigenerational condition of stress.

\section{Stress treatment}

Gestational/prenatal stress was applied daily from gestational days 12 through 18 . Restraint and swim stress were applied daily in a semi-random sequence at 8:30 am and 4:30 pm. This stress regimen corresponds to a mild to moderate stressor in rats [16]. Stress treatments were performed in a designated room other than the housing facility.

\section{Restraint}

Rats were placed in a transparent Plexiglas container $(5 \mathrm{~cm}$ inner diameter) for a period of 20 minutes each day [16]. The container had perforated ends to allow ventilation. The container maintained the animals in a standing position without compression of the body.

\section{Swim stress}

Rats were individually placed in a tub filled with water at room temperature water ( 22 degrees Celsius) for five minutes. The water was deep enough so that neither the rat's feet nor its tail had contact with the bottom. After the 5 minutes dams were towel dried and returned to their home cage.

\section{Antepartum behavioural analysis}

Behaviour was videorecorded using an infrared video surveillance system (Panasonic WV-BP330, Panasonic, Japan). Animals were monitored by the surveillance system starting at 48 hours prior to expected delivery. Behavioural observations were performed for 60-minute intervals from video recorded data. The scoring intervals included the periods of 14-15, 18-19, and 22-23 hours prior to parturition of the first pup. These intervals were chosen because they reveal the most significant changes in maternal behaviour among control rats. The amount of time spent engaged in tail chasing, and the total number of rotations performed were measured.

Initiation of tail chasing behaviour was scored when the dam took interest in her tail followed by chasing or holding the tail with the mouth. Completion of a tail chasing event was scored once the rat disengaged with her tail and initiated a different activity. Figure $2 \mathrm{~A}-\mathrm{C}$ illustrates a characteristic sequence of tail chasing behaviour. Tail grooming behaviour was not included in this analysis.

\section{Statistical analysis}

Statistical analysis was performed using a Statview software package (Abacus Concepts, CA, USA). All data were subjected to square root transformation to assure constant variance and Gaussian distribution prior to statistical testing. The data were subject to analysis of variance (ANOVA) for measures across all testing groups followed by post-hoc Fisher's PLSD test for differences between groups. Paired comparisons between time intervals were performed using paired $t$-tests. Correlation analysis was performed using a Fisher's $\mathrm{r}$ to $\mathrm{z}$ transformation. A $p$-value of less than 0.05 was chosen as the significance level for all statistical analyses. All data are presented as the mean \pm standard error (S.E.M.).

\section{Results}

Qualitative analysis: prenatal and transgenerational stress disrupt the patterns of tail chasing behaviour

The effect of prenatal and transgenerational stress on pregnant dams was noticeable from an observational perspective. Non-stress control animals were found to rotate within a specific sequence of events that comprise a departure (Figure 2A), engagement (Figure 2B) and return component (Figure 2C). Typically, a tail chasing event was initiated when a pregnant dam departed from her core nest area and began to engage with her tail (Figure 2A). While engaged with her tail, a dam would usually chase the tail, eventually pick it up and carry it in her mouth (Figure 2B). The dam's head movement towards the tail usually initiates ipsiversive horizontal rotational movements of the body that are accompanied by coordinated fore- and hind limb steps. Once the dam caught the 


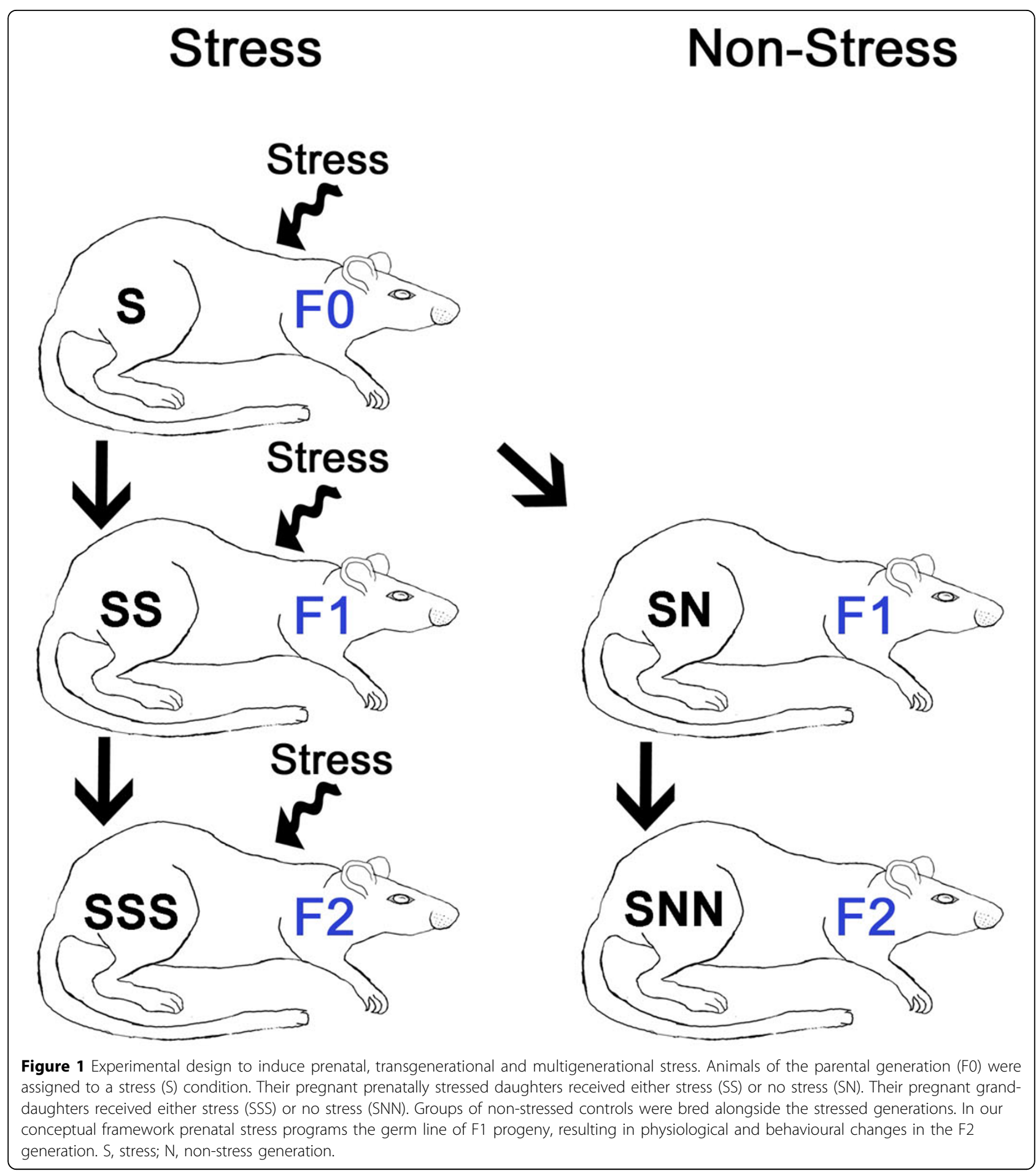

middle portion or tip of her tail she would carry the tail to her nest with the mouth and drop it at the core nest area (Figure 2C). Prenatal and transgenerational stress disrupted this characteristic sequence of events in that the dam showed reduced interest in her tail and frequently failed to show the departure, engagement and return components of this behaviour.
Quantitative analysis: prenatal and transgenerational stress reduces the frequency of tail chasing behaviour Antepartum tail chasing was assessed for time spent tail chasing and total number of rotations prior to parturition.

As displayed in Figure 3A the time spent in tail chasing showed a Group effect $(F(4,24)=5.44, p \leq 0.01$, power of 0.94$)$. There was no effect of gestational stress in the 
B1

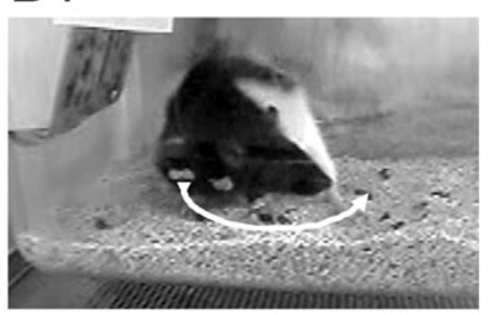

B2

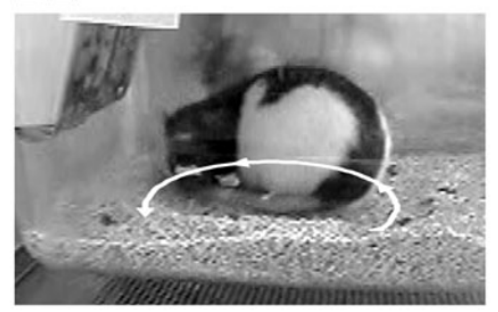

B3
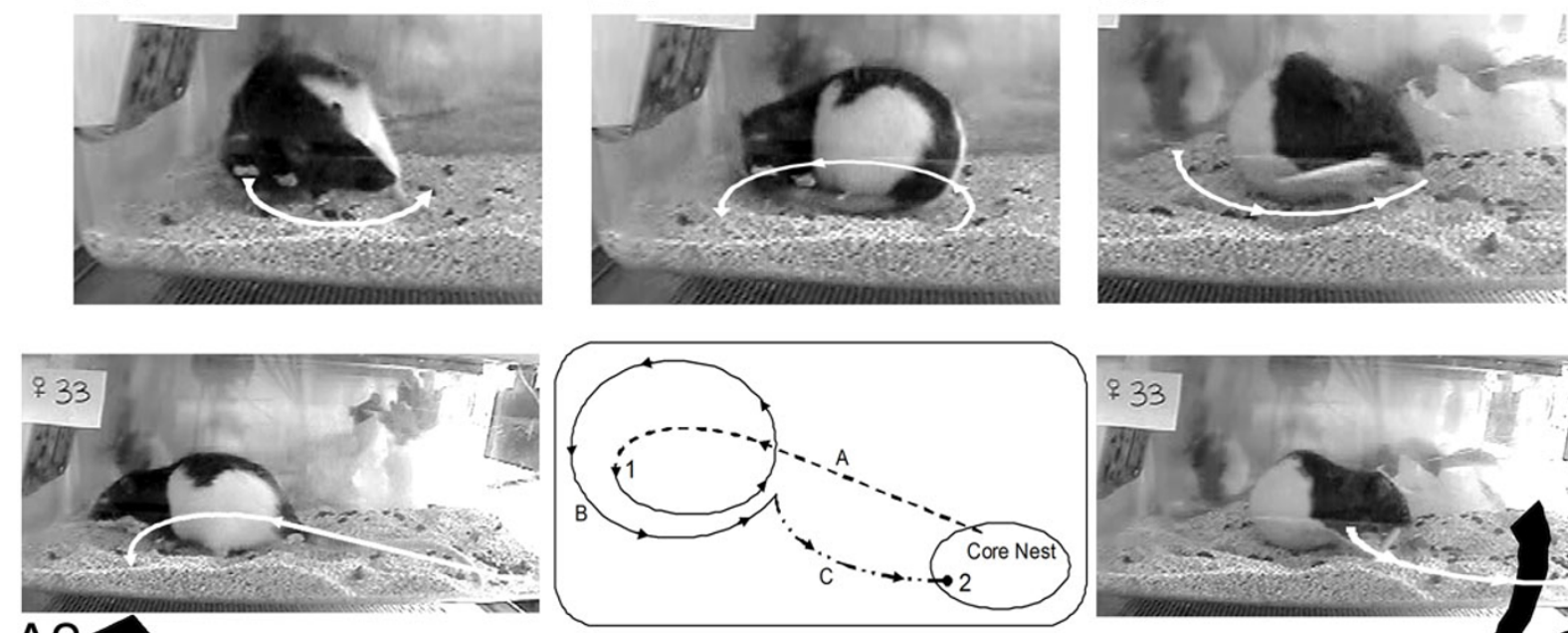

A2
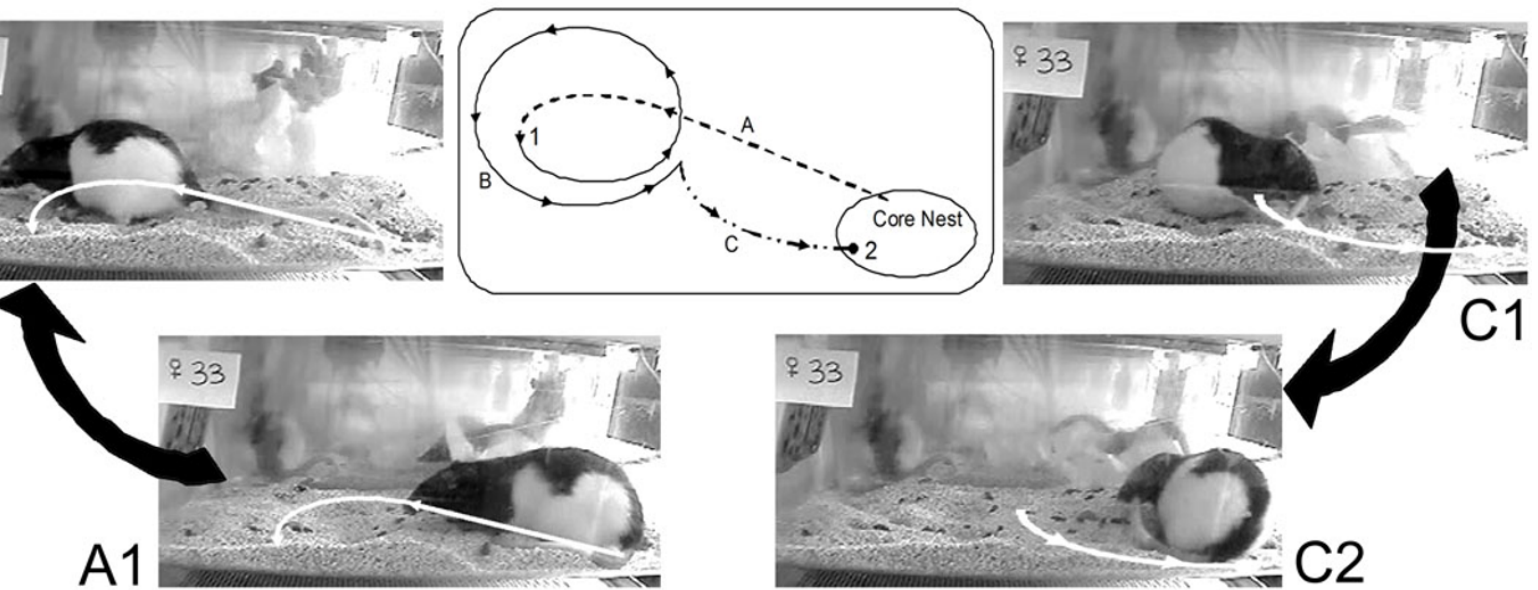

C1

Figure 2 Illustration of a representative sequence of movements comprising tail chasing in a naïve pregnant dam. Photographs in A represent departure from the core nest area. Photographs in B represent engagement with the tail. Photographs in $C$ represent a return to the core nest area. A1) Dam proceeds from core nest, A2) to the open space of her home cage. B1) Dam takes interest in her tail, B2) and rotates in the attempt to catch her tail with the mouth, B3) continuing the rotation while holding the tail in her mouth. C1) Dam with tail in mouth begins return to core nest, $\mathrm{C2}$ ) and delivers the tail to the core nest. The central map shows a representative trajectory of a horizontal movement sequence in the home cage. Numbers 1 and 2 indicate start and end points of the movement, respectively.

parental (F0) generation ( $\mathrm{p}=0.43)$. F1-SS prenatally and gestationally stressed dams spent significantly less time engaged in tail chasing behaviour $(\mathrm{p} \leq 0.01)$. Furthermore, the F2-SNN animals showed a significant reduction in the time spent in tail chasing behaviours when compared to controls $(\mathrm{p} \leq 0.01)$. The greatest effect was exhibited by the F2-SSS group, in which rotation was absent $(\mathrm{p} \leq 0.01)$, suggesting a cumulative effect of multigenerational stress. Also, there also was a significant reduction in F1-SS and F2-SSS when compared to parental F0-S rats ( $\mathrm{p} \leq 0.05$ and $\mathrm{p} \leq 0.01$, respectively).

Furthermore, the number of rotations also showed a significant effect of Group $(F(4,24)=5.27, p \leq 0.01$, power of $0.91)$. There was no difference between stressed and nonstressed F0 dams ( $\mathrm{p}=0.38)$. F1-SS animals performed fewer rotations than control animals $(\mathrm{p} \leq 0.01$; Figure $3 \mathrm{~B})$. In addition, F2-SNN dams $(\mathrm{p} \leq 0.01)$ and F2-SSS dams $(\mathrm{p} \leq 0.01)$ performed fewer rotations than control dams. There was a significant effect of generation because F1-SS, F2-SNN and F2-SSS rotated less than parental F0-S rats (all p's $\leq 0.05$ ).
Correlation analysis revealed that neither time spent tail chasing (weight gain: $r=0.077$; litter size: $r=0.173$ ) nor the number of rotations (weight gain: $r=0.071$; litter size: $\mathrm{r}=0.145$ ) were related to maternal weight gain or litter size.

The time course of changes from interval 23, 19 and 15 hours revealed a main effect of Group $(F(4,24)=5.82$, $\mathrm{p} \leq 0.01 ; \mathrm{F}(2,24)=4.86, \mathrm{p} \leq 0.01)$, Interval $(\mathrm{F}(2,24)=14.48$, $\mathrm{p} \leq 0.001 ; \mathrm{F}(2,24)=14.77, \mathrm{p} \leq 0.001)$ and a Group by Time interaction $(F(8,48)=3.75, p \leq 0.01 ; F(8,48)=4.19, p \leq 0.001)$ for the time spent tail chasing and the number of rotations, respectively. Control dams spent significantly more time chasing their tail at 19 hours $(\mathrm{t}=2.98, \mathrm{p} \leq 0.05)$ and 15 hours $(\mathrm{t}=4.49, \mathrm{p} \leq 0.01)$ prior to parturition compared to the 23-hour time point (Figure 4A). They also performed more rotations at 15 hours than at 23 hours prior to parturition $(t=2.91, p \leq 0.05$; Figure 4B). The time of F0-S dams increased from 19 to 15 hours $(\mathrm{t}=2.57, \mathrm{p} \leq 0.05)$. There was no change in tail chasing behaviour in F2 or F3 animals. 


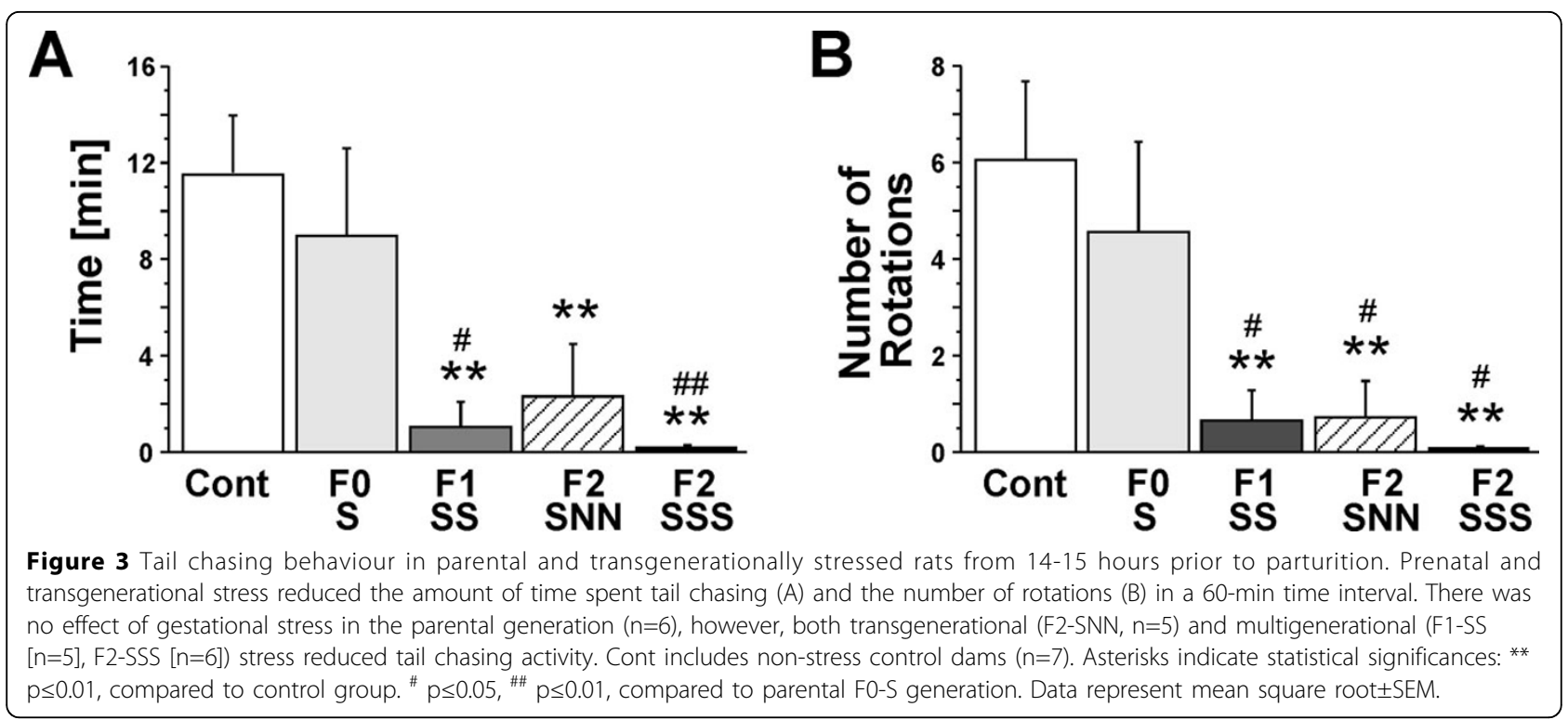

\section{Discussion}

The purpose of this study was to determine behavioural changes that occur prior to parturition as a result of gestational, prenatal and transgenerational stress. Using tail chasing as a new measure of antepartum maternal behaviour, we show that prenatal and transgenerational stress, but not gestational stress, alters this behaviour. We observed a significant decrease in the amount of tail chasing over that of controls during the antepartum period in F1 and F2 mothers that had experienced prenatal stress. We show that the effects of prenatal stress as expressed by reduced tail chasing are passed on to the next generation and its progeny. The effects of prenatal stress persist in the absence of stress in the filial generations, suggesting physiological and behavioural programming in the offspring with possibly lifelong consequences.

To date there has been very little investigation of rodent antepartum behaviours. We propose that maternal behaviour during the antepartum period may reflect preparatory activities, such as nest building. A previous study showed that nest building activities of the dam undergoes a significant increase during the 24 hours prior to parturition [17], which is in agreement with the time course of tail chasing behaviour. In the present study, however, dams were not provided with nest building material for better visibility during video analysis and thus no unequivocal correlations between tail chasing and nest building activities are possible. It is possible that the antepartum increase
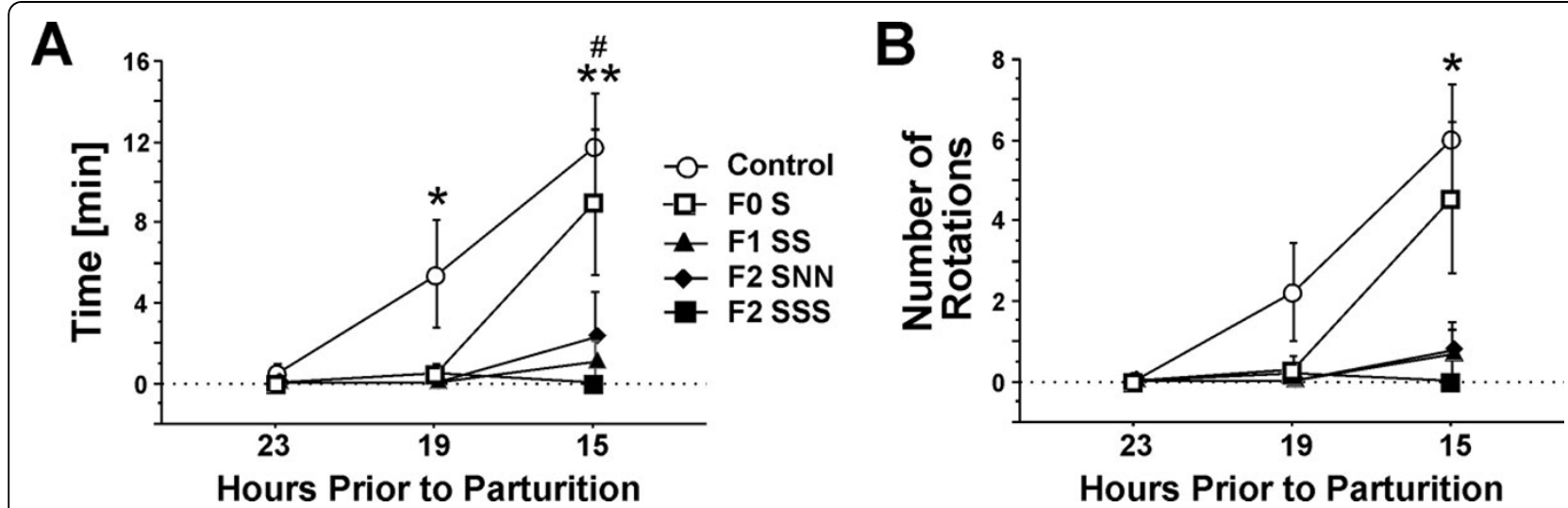

Figure 4 Time course of time spent tail chasing (A) and the number of rotations performed (B) in parental and transgenerationally stressed rats at 23, 19, and 15 hours prior to parturition. Tail chasing in control $(n=7)$ and parental FO-S $(n=6)$ dams increased from 23 to 15 hours prior to parturition. Note that there was no increase in tail chasing behaviours in transgenerational (F2-SNN, $n=5)$ and multigenerational (F1-SS [ $n=5$ ], F2-SSS [ $n=6]$ ) stressed animals. Asterisks indicate statistical significances: ${ }^{*} p \leq 0.05$ and ${ }^{* *} p \leq 0.01$ in control dams, ${ }^{*} p \leq 0.05$ in F0-S dams, compared to the 23 -hour time interval. Data represent mean square root \pm SEM. 
in tail chasing behaviour is indicative of post-partum maternal care. Maternal care, including licking and grooming as a form of tactile stimulation, has been shown to reduce the behavioural and endocrine consequences of preterm birth or early environmental adversity in rodents and human infants [18-21]. Tail chasing may also be indicative of other forms of maternal care, such as the retrieval of pups. The observation that the pregnant dam typically engages with the tail outside of her core nest area and completes the tail chasing bout by carrying it back to her nest would supports this hypothesis. Although the specific function of maternal tail chasing behaviour remains to be determined, the present findings suggest that antepartum maternal behaviour may represent a valid indicator of post-partum maternal care.

Periparturitional maternal behaviours may be particularly sensitive to the effects of stress. Prenatal stress may permanently alter brain development, which may manifest in altered nest building behaviour and behavioural simplification when a prenatally stressed rat matures and becomes pregnant [22]. Furthermore, corticosterone levels in pregnant rats peak on gestational day 18 and remain high until parturition [23]. The intricate endocrine changes of gestation and the rise in antepartum corticosterone levels in particular may stimulate central dopaminergic systems and lead to greater locomotor activity [24]. Thus, greater engagement in tail chasing behaviour in dams may be causally related to enhanced hypothalamopituitary-adrenal (HPA) axis activity preparing for parturition. It is possible, however, that HPA axis programming by prenatal and transgenerational stress reduces overall motor activity and leads to reduced tail chasing behaviour [25]. Furthermore, prenatal stress may alter basal activity of the HPA axis and the response to stress in adulthood $[1,26]$. The resulting imbalance of glucocorticoid-regulated endocrine factors participating in parturition may contribute to altering antepartum maternal behaviours.

Altered maternal behaviour during gestation may also reflect changes in profiles of progesterone levels in rodents. In rats, parturition is associated with a decrease in progesterone production, also termed progesterone withdrawal [27]. Progesterone plasma levels usually begin to decline on gestational day 19 [28]. In the present study, tail chasing behaviour was analyzed on gestational day 22 , 23, 19 and 15 hours prior to parturition, at a time of low progesterone levels. The time course of tail chasing behaviour in the 24 hours leading to parturition suggest that endocrine changes may mediate an increase or decrease in this activity. While gestational stress in the parental generation did not affect tail chasing behaviour and likely did not affect progesterone levels, prenatal and transgenerational stress may have diminished the engagement in tail chasing through interference with progesterone regulation and other components. This notion is supported by a study showing that the onset of maternal nest building at or about the end of pregnancy is associated with a fall in circulating levels of progesterone in rabbits [29]. Furthermore, inadequacy or absence of a nest area can adversely affect maternal care [29]. Notably, gestational stress can dysregulate progesterone formation in juvenile offspring [30], a change that may persist into adulthood in female F1 and F2 animals to perturb physiological and behavioural adjustments to pregnancy. If any of these changes contributed to the present behavioural observations, our data show that the underlying endocrine processes were not associated with profound maternal weight or litter size effects.

The influence of transgenerational programming by prenatal stress was evident in the F2-SNN generation. Although an F3 generation would be necessary to confirm truly epigenetic effects [31,32], our findings suggest that programming by prenatal stress disrupts tail chasing behaviour in the grand-offspring and great-grand-offspring. These effects may be due to direct germ line exposure to maternal stress in the womb. Moreover, the exposure to multi-generational stress in F1-SS and F2SSS rats indicates that prenatal stress has cumulative, context-dependent consequences. These findings suggest that epigenetic mechanisms may mediate a gradually altering physiological response to recurrent stress in each generation. The formation of an epigenetic memory to a single or recurrent adverse event within a family history may assist in adjusting physiological and/or behavioural patterns to a stressful environment. Epigenetic memory refers to transgenerationally stable, yet dynamic re-programming of the germline epigenome that transfers information across generations in the absence of changes in DNA sequence [33,34]. Through this kind of memory, the trait of altered maternal behaviour may be passed on to the subsequent generation $[13,35,36]$ via, for example, a heritable pattern of hypermethylation of the gene encoding brain-derived neurotrophic factor (BDNF) [37]. In the offspring, the resulting reduction in BDNF expression and low levels of this growth factor in the prefrontal cortex during development may have drastic consequences for cognitive and affective abilities and the response to stress in adulthood.

In conclusion, our findings show that antepartum maternal behaviour may have particularly predictive value of an activated stress response, parturition and post-partum maternal care towards her offspring. Importantly, prenatal stress may program physiological and behavioural responses to pregnancy and postpartum maternal care in subsequent generations and their progeny.

Authors' contributions

IDW, FCRZ, DMO, KB and GAM designed the study. IDW, FCRZ, JCR, and EAF performed the experiments, collected and analyzed the data. IDW and GAM 
completed the statistical analysis and prepared the figures. IDW, FCRZ, KB and GAM wrote the manuscript.

\section{Competing interests}

The authors declare no competing financial interests.

\section{Acknowledgements}

This research was supported by Preterm Birth and Healthy Outcomes Team (PreHOT) funded by the Alberta Innovates-Health Solutions Interdisciplinary Team Grant \#200700595 (DO, KB, GM), Hotchkiss Brain Institute (FZ), Norlien Foundation (FZ), and the Canadian Institutes of Health Research (GM). GM is a Senior Scholar of Al-HS.

\section{Declarations}

This article has been published as part of BMC Pregnancy and Childbirth Volume 13 Supplement 1, 2013: Preterm Birth: Interdisciplinary Research from the Preterm Birth and Healthy Outcomes Team (PreHOT). The full contents of the supplement are available online athttp://www. biomedcentral.com/bmcpregnancychildbirth/supplements/13/S1. All of the publication fees will be funded by the Preterm Birth and Healthy Outcomes Team Interdisciplinary Team Grant (\#200700595) from Alberta Innovates - Health Solutions, formerly the Alberta Heritage Foundation for Medical Research.

\section{Author details}

${ }^{1}$ Canadian Centre for Behavioural Neuroscience, University of Lethbridge, 4401 University Drive, Lethbridge, AB, Canada T1K3M4. ${ }^{2}$ Departments of Obstetrics \& Gynecology, Pediatrics and Physiology, University of Alberta, 227 HMRC, University of Alberta, Edmonton, AB, Canada T6G2S2. ${ }^{3}$ Faculty of Nursing, University of Calgary, 2500 University Dr. NW, Calgary, AB, Canada T2N1N4.

Published: 31 January 2013

\section{References}

1. Harris A, Seckl J: Glucocorticoids, prenatal stress and the programming of disease. Horm Behav 2011, 59:279-289.

2. Treyvaud K, Anderson VA, Howard K, Bear M, Hunt RW, Doyle LW, Inder TE, Woodward L, Anderson PJ: Parenting behavior is associated with the early neurobehavioral development of very preterm children. Pediatrics 2009, 123:555-561.

3. Van Hasselt FN, Tieskens JM, Trezza V, Krugers HJ, Vanderschuren $\sqcup$, Joëls M: Within-litter variation in maternal care received by individual pups correlates with adolescent social play behavior in male rats. Physiol Behav 2012, 106:701-706.

4. Francis D, Diorio J, Liu D, Meaney MJ: Nongenomic transmission across generations of maternal behavior and stress responses in the rat. Science 1999, 286:1155-1158.

5. Fish EW, Shahrokh D, Bagot R, Caldji C, Bredy T, Szyf M, Meaney MJ: Epigenetic programming of stress responses through variations in maternal care. Ann N Y Acad Sci 2004, 1036:167-180.

6. Parent $\mathrm{Cl}$, Del Corpo A, Cameron NM, Meaney MJ: Maternal care associates with play dominance rank among adult female rats. Dev Psychobiol 2012, doi: 10.1002/dev.21070.

7. Murgatroyd CA, Nephew BC: Effects of early life social stress on maternal behavior and neuroendocrinology. Psychoneuroendocrinology 2012, [Epub ahead of print].

8. Champagne FA, Meaney MJ: Stress during gestation alters postpartum maternal care and the development of the offspring in a rodent model. Biol Psychiatry 2006, 59:1227-1235.

9. Matthews SG, Phillips DI: Transgenerational inheritance of stress pathology. Exp Neurol 2012, 233:95-101

10. Crews D, Gillette R, Scarpino SV, Manikkam M, Savenkova MI, Skinner MK: Epigenetic transgenerational inheritance of altered stress responses. ProC Natl Acad Sci 2012, 109:9143-9148.

11. Fleming AS, Kraemer GW, Gonzalez A, Lovic V, Rees S, Melo A: Mothering begets mothering: the transmission of behavior and its neurobiology across generations. Pharmacol Biochem Behav 2002, 73:61-75.

12. Champagne FA, Francis DD, Mar A, Meaney MJ: Variations in maternal care in the rat as a mediating influence for the effects of environment on development. Physiol Behav 2003, 79:359-371.
13. Weaver IC, Cervoni N, Champagne FA, D'Alessio AC, Sharma S, Seckl JR, Dymov S, Szyf M, Meaney MJ: Epigenetic programming by maternal behavior. Nature Neurosci 2004, 7:847-854.

14. Skinner MK, Manikkam M, Guerrero-Bosagna C: Epigentic transgenerational actions of enviornmnental factors in disease etiology. Trends Endocrinol 2010, 21:214-222.

15. Zucchi FCR, Yao Y, Oliveira DJ, Inntskyy Y, Olson DM, Benzies K, Kovalchuk I, Kovalchuk O, Metz GA: Maternal stress programs offspring emotional state and miRNA expression as markers of psychiatric and neurological disease. PlOS ONE 2012, in press.

16. Metz GA, Jadavji NM, Smith LK: Modulation of motor function by stress: a novel concept of the effects of stress and corticosterone on behavior. Eur J Neurosci 2005, 22:1190-1200.

17. Denenberg $V$, Taylor R, Zarrow M: Maternal behavior in the rat: an investigation and quantification of nest building. Behaviour 1969, 34:1-16.

18. Pauk J, Kuhn CM, Field TM, Schanberg SM: Positive effects of tactile versus kinesthetic or vestibular stimulation on neuroendocrine and ODC activity in maternally-deprived rat pups. Life Sci 1986, 39:2081-2087.

19. Kuhn CM, Schanberg SM, Field T, Symanski R, Zimmerman E, Scafidi F, Roberts J: Tactile-kinesthetic stimulation effects on sympathetic and adrenocortical function in preterm infants. J Pediatr 1991, 119:434-440.

20. Caldji C, Tannenbaum B, Sharma S, Francis D, Plotsky PM, Meaney MJ: Maternal care during infancy regulates the development of neural systems mediating the expression of fearfulness in the rat. Proc Natl Acad Sci USA 1998, 95:5335-5340.

21. Sharp H, Pickles A, Meaney M, Marshall K, Tibu F, Hill J: Frequency of infant stroking reported by mothers moderates the effect of prenatal depression on infant behavioural and physiological outcomes. PLOS One 2012, 7:e45446.

22. Whishaw IQ, Metz GA, Kolb B, Pellis SM: Accelerated nervous system development contributes to behavioral efficiency in the laboratory mouse: a behavioral review and theoretical proposal. Dev Psychobiol 2001, 39(3):151-170.

23. Dupouy JP, Coffigny $H$, Magre S: Maternal and foetal corticosterone levels during late pregnancy in rats. J Endocrinol 1975, 65(3):347-352.

24. Piazza PV, Rouge'-Pont F, Deroche V, Maccari S, Le Moal M, Simon H: Suppression of glucocorticoid secretion and antipsychotic drugs have similar effects on the mesolimbic dopaminergic transmission. Pro. Natl Acad Sci USA 1996, 93:8716-8720.

25. Metz GA: Stress as a modulator of motor system function and pathology. Rev Neurosci 2007, 18(3-4):209-222.

26. Glover $\mathrm{V}, \mathrm{O}^{\prime}$ Connor TG, O'Donnell K: Prenatal stress and the programming of the HPA axis. Neurosci Biobehav Rev 2010, 35(1):17-22.

27. Mitchell BF, Taggart MJ: Are animal models relevant to key aspects of human parturition? Am J Physiol Regul Integr Comp Physiol 2009, 297:525-545

28. Puri CP, Garfield RE: Changes in hormone levels and gap junctions in the rat uterus during pregnancy and parturition. Biol Reprod 1982, 27:967-975.

29. Zarrow MX, Sawin PB, Ross S, Denenberg VH, Crary D, Wilson DE, Farooq A: Maternal Behaviour in the Rabbit: Evidence for an Endocrine Basis of Maternal Nest Building and Additional Data on Maternal Nest Building in the Dutch-Belted Race. J Reprod Fertil 1961, 2:152-162.

30. Paris JJ, Frye CA: Juvenile offspring of rats exposed to restraint stress in late gestation have impaired cognitive performance and dysregulated progestogen formation. Stress 2011, 14(1):23-32.

31. Skinner MK: What is an epigenetic transgenerational phenotype? F3 or F2. Reprod Toxicol 2008, 25(1):2-6.

32. Zucchi FC, Yao Y, Metz GA: The secret language of destiny: stress imprinting and transgenerational origins of disease. Front Genet 2012, 3:96.

33. Zambrano E: The transgenerational mechanisms in developmental programming of metabolic diseases. Rev Invest Clin 2009, 61(1):41-52.

34. Migicovsky Z, Kovalchuk I: Epigenetic memory in mammals. Front Genet 2011, 2:28.

35. Weaver IC, D'Alessio AC, Brown SE, Hellstrom IC, Dymov S, Sharma S, Szyf M, Meaney MJ: The transcription factor nerve growth factorinducible protein a mediates epigenetic programming: altering epigenetic marks by immediate-early genes. J Neurosci 2007, 27:1756-1768.

36. Cameron NM: Maternal programming of reproductive function and behavior in the female rat. Front Evol Neurosci 2011, 3:10. 
37. Roth $T L$, Lubin FD, Funk AJ, Sweatt JD: Lasting epigenetic influence of early life adversity on the BDNF gene. Biol Psychiatry 2009, 65(9):760-769.

doi:10.1186/1471-2393-13-S1-S9

Cite this article as: Ward et al:: Transgenerational programming of

maternal behaviour by prenatal stress. BMC Pregnancy and Childbirth 2013 13(Suppl 1):S9.

Submit your next manuscript to BioMed Central and take full advantage of:

- Convenient online submission

- Thorough peer review

- No space constraints or color figure charges

- Immediate publication on acceptance

- Inclusion in PubMed, CAS, Scopus and Google Scholar

- Research which is freely available for redistribution

Submit your manuscript at www.biomedcentral.com/submit 